\title{
ON CONVERSION OF RESONANT PROBLEM TO NON-RESONANT ONE
}

\author{
N. SVEIKATE AND F. SADYRBAEV
}

Received 01 May, 2016

\begin{abstract}
We consider a nonlinear resonant boundary value problem. To prove the existence of a solution to a given boundary value problem we replace the linear part of a given equation by non-resonant linear part. First, to modify a resonant problem to a regular one we use the Taylor expansion for $f\left(t, x, x^{\prime}\right)$ with respect to $x$. The second way of conversion a given problem to nonresonant one is based on an appropriate choice of "good" approximation to expected solution. We provide the existence results illustrating both ways.
\end{abstract}

2010 Mathematics Subject Classification: 34B10; 34B15

Keywords: resonant nonlinear boundary value problem, quasilinearization approach

\section{INTRODUCTION}

When studying two-point boundary value problems for a nonlinear second order equation

$$
x^{\prime \prime}=f\left(t, x, x^{\prime}\right)
$$

we first look at the right side. If $f$ is continuous and bounded (in modulus) function then the problem is solvable if the respective homogeneous problem

$$
x^{\prime \prime}=0, \quad \text { +boundary conditions }
$$

has only the trivial solution. Otherwise the problem may be unsolvable and this depends on the right hand side of an equation.

Similarly, if we consider the problem with the linear part

$$
\begin{gathered}
x^{\prime \prime}+p(t) x^{\prime}+q(t) x=F\left(t, x, x^{\prime}\right), \\
x(a)=A, \quad x(b)=B,
\end{gathered}
$$

we are sure that the problem is solvable (for $F$ bounded) if the respective homogeneous problem

$$
x^{\prime \prime}+p(t) x^{\prime}+q(t) x=0, \quad x(a)=0, x(b)=0
$$


has only the trivial solution. If the above problem has a nontrivial solution also, then the following question arises: which right hand sides $F\left(t, x, x^{\prime}\right)$ are "good" and which are "bad" for the existence of a solution.

The answer is given by the Fredholm theory for $F=F(t)$ : if the right hand side is "orthogonal" to an eigenfunction of a conjugate linear part, then this right side function $F(t)$ is "good".

The problem (1.3) with the property that the respective homogeneous problem has a nontrivial solution, is called resonant. There is intensive literature on solvability of resonant problems. Usually the appropriate conditions on the linear part and on the nonlinearity are imposed in order the problem to be solvable. One may consult recent articles $[1,5,12]$ and references therein for the respective bibliography.

In the paper [9] a resonant problem for the second order nonlinear equation is treated from "inside". First the type of a solution is introduced. It is shown afterwards that a resonant problem that has a solution of definite type in fact can be reduced to a non-resonant problem with the similar type linear part.

In this note we would like to analyze this process of conversion of a resonant problem to non-resonant one in details. It is to be mentioned that the natural idea of "a shift" from a resonant linear part to a non-resonant one was employed in the papers $[4,5,13]$. "Shift" arguments in a broad sense (replacing the linear part of a given equation multiply by different non-resonant linear parts and proving the existence of multiple solutions to a given boundary value problem) were applied for the study of resonant problems in [12] in context of the quasi-linearization approach [3]. In Section 4 of this paper we consider two ways of relaxing the quasilinearization arguments used in [12].

In order to make exposition self-contained first we introduce notions of the type of a linear part of an equation and that of the type of a solution.

\section{TYPES OF LINEAR PARTS AND TYPES OF SOLUTIONS}

Consider quasi-linear problem (1.3), (1.4). We say that the linear part $\left(l_{2} x\right)(t)=$ $x^{\prime \prime}+p(t) x^{\prime}+q(t)$ has type $i$, if a solution $y(t)$ of the Cauchy problem

$$
y^{\prime \prime}+p(t) y^{\prime}+q(t) y=0, \quad y(a)=0, y^{\prime}(a)=1
$$

has exactly $i$ zeros in the interval $(a, b)$ and $y(b) \neq 0$.

If $y(b)=0$ then the linear part $\left(l_{2} x\right)(t)$ is resonant.

For instance, the linear part $x^{\prime \prime}+k^{2} x$, where $3 \pi<k<4 \pi$, has type 3 .

Let us pass to definition of the type of a solution.

Let $\xi(t)$ be a solution of the BVP

$$
x^{\prime \prime}=f\left(t, x, x^{\prime}\right), \quad x(a)=A, x(b)=B .
$$

The right hand side function $f$ may contain a linear part. 
Consider the respective equation of variations

$$
y^{\prime \prime}=f_{x}\left(t, \xi(t), \xi^{\prime}(t)\right) y+f_{x^{\prime}}\left(t, \xi(t), \xi^{\prime}(t)\right) y^{\prime} .
$$

Definition 1. Let $\xi(t)$ be a solution of BVP. We say that the type of $\xi(t)$ is $i$, if equation of variations (2.3) with respect to $\xi(t)$ is such that a solution $y(t)$ with the initial conditions

$$
y(a)=0, \quad y^{\prime}(a)=1
$$

has exactly $i$ zeros in the interval $(a, b)$ and $y(b) \neq 0$. Denote this:

$$
\operatorname{type}(\xi)=i \text {. }
$$

If moreover $y(b)=0$, denote the intermediate type

$$
\text { type }(\xi)=(i, i+1) \text {. }
$$

Remark 1. Therefore, a solution of type $(i, i+1)$ is a solution $\xi(t)$ such that the respective $y(t)$ has exactly $i$ zeros in $(a, b)$ and $y(b)=0$.

Remark 2. The study of solutions of BVP in terms of solutions of equations of variations was initiated in early papers [6-8]. The authors have observed the existence of solutions of zero type (in our terminology) in problems satisfying some standard requirements.

Example 1. The trivial solution $\xi(t) \equiv 0$ of the problem $x^{\prime \prime}=-x+x^{3}, x(0)=$ $0, x(\pi)=0$ is of type $(0,1)$ since a solution $y(t)$ of the Cauchy problem $y^{\prime \prime}=-y$ (equation of variations with respect to $\xi$ ), $y(0)=0, y^{\prime}(0)=1$ has the first zero at $t=\pi$.

\section{REDUCTION TO QUASI-LINEAR PROBLEM}

Return to the problem (1.1), (1.4). The right side $f$ may contain linear part. The case of the problem (1.1), (1.4) being resonant is not excluded.

Theorem 1. Suppose the problem (1.1), (1.4) has a solution $\xi(t)$ of type $i$.

Then the problem can be reformulated in the quasi-linear form (1.3), (1.4), where the linear part $x^{\prime \prime}+p(t) x^{\prime}+q(t) x$ in (1.3) has the type $i$.

Proof. One has that

$$
\begin{aligned}
x^{\prime \prime} & -\xi^{\prime \prime}(t)=f\left(t, x, x^{\prime}\right)-f\left(t, \xi(t), \xi^{\prime}(t)\right) \\
& =f_{x}\left(t, \xi(t), \xi^{\prime}(t)\right)(x-\xi(t))+f_{x^{\prime}}\left(t, \xi(t), \xi^{\prime}(t)\right)\left(x^{\prime}-\xi^{\prime}(t)\right)+\varphi\left(t, x, x^{\prime}\right)
\end{aligned}
$$

or

$$
\begin{aligned}
& x^{\prime \prime}-f_{x^{\prime}}\left(t, \xi(t), \xi^{\prime}(t)\right) x^{\prime}-f_{x}\left(t, \xi(t), \xi^{\prime}(t)\right) x \\
& =\xi^{\prime \prime}(t)-f_{x^{\prime}}\left(t, \xi(t), \xi^{\prime}(t)\right) \xi^{\prime}(t)-f_{x}\left(t, \xi(t), \xi^{\prime}(t)\right) \xi(t)+\varphi\left(t, x, x^{\prime}\right)
\end{aligned}
$$


and finally

$$
\left(l_{2} x\right)(t)=h(t)+\widetilde{\varphi}\left(t, x, x^{\prime}\right)
$$

where

$$
\begin{aligned}
\left(l_{2} x\right)(t) & =x^{\prime \prime}-f_{x^{\prime}}\left(t, \xi(t), \xi^{\prime}(t)\right) x^{\prime}-f_{x}\left(t, \xi(t), \xi^{\prime}(t)\right) x, \\
h(t) & =\xi^{\prime \prime}(t)-f_{x^{\prime}}\left(t, \xi(t), \xi^{\prime}(t)\right) \xi^{\prime}(t)-f_{x}\left(t, \xi(t), \xi^{\prime}(t)\right) \xi(t),
\end{aligned}
$$

$\widetilde{\varphi}\left(t, x, x^{\prime}\right)$ is a smooth $\left(C^{1}\right)$ bounded function which coincides with $\varphi\left(t, x, x^{\prime}\right)$ in some vicinity of $\left(t, \xi(t), \xi^{\prime}(t)\right), t \in[a, b]$.

The linear part $\left(l_{2} x\right)(t)$ has type $i$ since a solution $\xi(t)$ has type $i$. Function $\xi$, by construction, is a solution of BVP (3.3), (1.4).

Remark 3. The above reduction is not possible if a solution $\xi(t)$ is of indefinite type $(i, i+1)$.

\section{RESONANT PROBLEMS}

Consider, for simplicity, the problem

$$
x^{\prime \prime}+k^{2} x=f(t, x), \quad x(0)=0, \quad x(1)=0 .
$$

If $f$ is continuous function (together with $f_{x}$ ) and bounded, then the problem above is solvable provided that $k$ is not multiple of $\pi$. If $k=i \pi$, where $i$ is an integer, then the homogeneous problem

$$
x^{\prime \prime}+k^{2} x=0, \quad x(0)=0, \quad x(1)=0
$$

has a nontrivial solution and solvability of the problem (4.1) is not guaranteed.

The Fredholm alternative gives an answer in case $f=f(t)$. If not, the following approach was proposed. Change the left side in equation so that it is not resonant yet, add the same term to the right, and finally truncate modified right side appropriately obtaining nonresonant quasi-linear problem. This problem has a solution and if some estimates can be proved for a solution, then this solution may solve also the original problem.

Look how this works. Consider instead of (4.1) the equivalent problem

$$
x^{\prime \prime}+k^{2} x+\varepsilon^{2} x=f(t, x)+\varepsilon^{2} x, \quad x(0)=0, \quad x(1)=0 .
$$

Truncate the right side in equation (4.3) so that the truncated right side function $F(t, x)$ coincides with $f(t, x)+\varepsilon^{2} x$ for $x \in[-N, N]$ and $t \in[0,1]$.

The modified problem

$$
x^{\prime \prime}+k^{2} x+\varepsilon^{2} x=F(t, x), \quad x(0)=0, \quad x(1)=0
$$

has a solution $x(t)$ and the representation

$$
x(t)=\int_{0}^{1} G(t, s) F(s, x(s)) d s
$$


is valid, where $G$ is the Green's function associated with new (nonresonant) left side. If the key inequality

$$
\Gamma \cdot M \leq N
$$

holds, where $\Gamma$ and $M$ are respectively bounds (the estimate constants) for the Green's function $|G|$ and $|F(t, x)|$, then $|x(t)| \leq N$ and $x(t)$ is a solution of (4.1). Due to the results in [10] and [11], there is a solution $x(t)$ that has definite type induced by the linear part in (4.4). Therefore multiple application of this scheme using multiple different linear parts can prove the existence of multiple solutions of the (resonant) problem (4.1). This scheme was tested on equations of the Emden Fowler type in [12] (see also [3]).

In the sequel we would like to discuss two ways of relaxing inequality (4.5).

\subsection{Relaxing (4.5) - first way}

It is known (see [12]) that the above approach is applicable to resonant problems of the type

$$
x^{\prime \prime}+\pi^{2} x=-|x|^{p} \operatorname{sign} x, \quad x(0)=x(1)=0, \quad t \in[0,1] .
$$

The reason is, probably, that after modifying the equation in (4.6) looks

$$
x^{\prime \prime}+\pi^{2} x+\varepsilon^{2} x=-|x|^{p} \operatorname{sign} x+\varepsilon^{2} x
$$

and the right hand side function

$$
-|x|^{p} \operatorname{sign} x+\varepsilon^{2} x
$$

for appropriate choice of $\varepsilon$ is uniformly small on an interval $[-N, N]$. In other words, the right side of (4.6) is well approximated by a linear term $\varepsilon^{2} x$ in the interval $[-N, N]$. Then a constant $M$ in (4.5) is relatively small also and the inequality (4.5) is likely to be satisfied.

Let us extend this technique to more general cases. Consider the problem

$$
x^{\prime \prime}+\pi^{2} x=g(x), \quad x(0)=x(1)=0, \quad t \in[0,1] .
$$

It follows from the Taylor expansion that

$$
g(x)-g\left(x_{0}\right) \simeq g^{\prime}\left(x_{0}\right)\left(x-x_{0}\right) .
$$

Adding $-g\left(x_{0}\right)-g^{\prime}\left(x_{0}\right)\left(x-x_{0}\right)$ to both sides, one obtains

$$
x^{\prime \prime}+\pi^{2} x-g\left(x_{0}\right)-g^{\prime}\left(x_{0}\right)\left(x-x_{0}\right)=g(x)-g\left(x_{0}\right)-g^{\prime}\left(x_{0}\right)\left(x-x_{0}\right) .
$$

The right hand side is small if an expected solution $x(t)$ is close to a constant $x_{0}$. Suppose $x_{0}=0$ and assume we are looking for solutions with small amplitude of oscillations. Equation (4.11) becomes

$$
x^{\prime \prime}+\pi^{2} x-g(0)-g^{\prime}(0) x=g(x)-g(0)-g^{\prime}(0) x
$$

or, provided that $g(0)=0$,

$$
x^{\prime \prime}+\pi^{2} x-g^{\prime}(0) x=g(x)-g^{\prime}(0) x .
$$


Example 2. $g(x)=-k \arctan x$

Consider the boundary value problem

$$
x^{\prime \prime}+\pi^{2} x=-k \arctan x, x(0)=x(1)=0 .
$$

Reduce it to the form (4.13)

$$
\begin{gathered}
x^{\prime \prime}+\pi^{2} x-g^{\prime}(0) x=g(x)-g^{\prime}(0) x, \\
x^{\prime \prime}+\pi^{2} x+\left.\frac{k}{1+x^{2}}\right|_{x=0} x=-k \arctan x+\left.\frac{k}{1+x^{2}}\right|_{x=0} x, \\
x^{\prime \prime}+\pi^{2} x+k x=-k \arctan x+k x .
\end{gathered}
$$

We obtain the differential equation

$$
x^{\prime \prime}+\left(\pi^{2}+k\right) x=-k \arctan x+k x,
$$

where the linear part is no more resonant with respect to the given boundary conditions.

The appropriate estimate of the Green's function of the corresponding homogeneous boundary value problem is a constant

$$
\Gamma=\frac{1}{\sqrt{\pi^{2}+k}\left|\sin \sqrt{\pi^{2}+k}\right|} .
$$

We define $N>0$ and $M(N)=|-k \arctan N+k N|$.

Therefore if the inequality

$$
\frac{|-k \arctan N+k N|}{\sqrt{\pi^{2}+k}\left|\sin \sqrt{\pi^{2}+k}\right|} \leq N
$$

holds, then the boundary value problem (4.14) has a solution $|x(t)| \leq N$.

For example, if $k=1$, then the inequality (4.16) holds for $N \leq 2.4$.

Example 3. $g(x)=-k \sin x$. Consider the boundary value problem

$$
x^{\prime \prime}+\pi^{2} x=-k \sin x, x(0)=x(1)=0 .
$$

Analogously, as in the previous example we reduce the equation (4.17) to the form (4.13). We obtain the differential equation

$$
x^{\prime \prime}+\left(\pi^{2}+k\right) x=-k \sin x+k x,
$$

where the linear part is no more resonant with respect to the given boundary conditions.

The estimate of the Green's function for the corresponding homogeneous boundary value problem is given by (4.15).

Analogously, we define $N$ positive and $M(N)=|-k \sin N+k N|$. 
Therefore if the inequality

$$
\frac{|-k \sin N+k N|}{\sqrt{\pi^{2}+k}\left|\sin \sqrt{\pi^{2}+k}\right|} \leq N .
$$

holds, then the boundary value problem (4.17) has a solution $|x(t)| \leq N$.

And, for example, if $k=1$, then the inequality (4.18) holds for $N \leq 1.92$.

Example 4. $g(x)=-k\left(e^{x}-1\right)$.

Consider the boundary value problem

$$
x^{\prime \prime}+\pi^{2} x=-k\left(e^{x}-1\right), x(0)=x(1)=0 .
$$

Analogously, as in the previous examples we reduce the equation (4.19) to the form (4.13). We obtain the differential equation

$$
x^{\prime \prime}+\left(\pi^{2}+k\right) x=-k\left(e^{x}-1\right)+k x,
$$

where the linear part is no more resonant with respect to the given boundary conditions.

The estimate of the Green's function for the corresponding homogeneous boundary value problem is given by (4.15).

Analogously, we define $N$ positive and $M(N)=\left|-k\left(e^{N}-1\right)+k N\right|$.

Therefore if the inequality

$$
\frac{\left|-k\left(e^{N}-1\right)+k N\right|}{\sqrt{\pi^{2}+k}\left|\sin \sqrt{\pi^{2}+k}\right|} \leq N
$$

holds, then the boundary value problem (4.19) has a solution $|x(t)| \leq N$. And, for example, if $k=1$, then the inequality (4.20) holds for $N \leq 0.77$.

\subsection{Relaxing (4.5) - second way}

The second way of relaxing the inequality (4.5) is based on an appropriate choice of "good" approximation to an expected solution.

Consider the (resonant) problem

$$
x^{\prime \prime}=f(t, x), \quad x(0)=x(1)=0, \quad t \in[0,1],
$$

where $f(t, x)=-\pi^{2} x+g(x)$ and $g(x)$ is a continuous generally nonlinear function.

Assume that there exists $u \in C^{2}([0,1])$ and $N \geq 0$ such that the estimate

$$
\Gamma \cdot M(N) \leq N
$$

holds, where $\Gamma$ is an estimate of the Green's function $G(t, s)(G$ is the Green's function for $x^{\prime \prime}=0, x(0)=x(1)=0$ and $M(N)=\max \left\{\left|f(t, x)-u^{\prime \prime}(t)\right|: 0 \leq t \leq\right.$ $1,|x-u(t)| \leq N\}$.

We will show that then the problem (4.21) has a solution. 
Function $u(t)$ plays the role of an approximation to a solution $x(t)$ of the problem (4.21). Introduce new variable $y=x-u(t)$. Define

$$
\begin{aligned}
f_{1}(t, x) & =f(t, x)-u^{\prime \prime}(t), \\
F_{1}(t, y) & =f_{1}(t, u(t)+y), \\
F(t, y) & =F_{1}(t, \delta(-N, y, N)),
\end{aligned}
$$

where $\delta(-N, y, N)$ is the "cut off" function. Notice that

$$
\max \{|F(t, y)|: 0 \leq t \leq 1,|y| \leq N\}=M(N) .
$$

Consider the problem

$$
y^{\prime \prime}=F(t, y) \quad y(0)=0, y(1)=0,
$$

which is solvable (by R. Conti's theorem, [2]). By virtue of (4.22) any solution $y(t)$ of (4.23) satisfies the estimate $|y(t)| \leq N \quad \forall t \in I$. Then $y^{\prime \prime}(t)=F_{1}(t, y(t))=$ $f_{1}(t, u(t)+y(t))=f(t, x(t))-u^{\prime \prime}(t)$ and the function $y(t)+u(t)$ solves the problem (4.21).

This technique will work out if the approximation $u(t)$ is close to a solution $x(t)$. Then $M(N)$ in (4.22) may be small for $|x-u(t)| \leq N$, where $N$ is small also.

This approach is illustrated by the examples below. First a solution $x(t)$ should be found numerically. Second, a solution $x(t)$ may be approximated by an appropriate function (the function $\alpha \sin \beta t$ will be tested) and all the estimations (4.22) are to be proved.

Example 5. $g(x)=-\arctan x$.

Consider the boundary value problem

$$
x^{\prime \prime}=-\pi^{2} x-\arctan x, x(0)=x(1)=0 .
$$

Denote $u(t)=32 \sin \pi t$. Introduce new variable $y=x-u(t)$. Therefore $x=y+$ $u(t)$.

Define

$$
\begin{aligned}
f_{1}(t, x) & =g(t, x)-u^{\prime \prime}(t)=-\pi^{2} x-\arctan x+32 \pi^{2} \sin \pi t, \\
F_{1}(t, y) & =f_{1}(t, u(t)+y) \\
& =-\pi^{2}(y+32 \sin \pi t)-\arctan (y+32 \sin \pi t)+32 \pi^{2} \sin \pi t \\
& =-\pi^{2} y-\arctan (y+32 \sin \pi t), \\
F(t, y) & =F_{1}(t, \delta(-N, y, N)) \\
& =-\pi^{2} \delta(-N, y, N)-\arctan (\delta(-N, y, N)+32 \sin \pi t),
\end{aligned}
$$

where $\delta(-N, y, N)$ is the "cut off" function.

Notice that

$$
\max \{|F(t, y)|: 0 \leq t \leq 1,|y| \leq N\}=M(N) .
$$


Consider the problem

$$
y^{\prime \prime}=F(t, y), \quad y(0)=0, \quad y(1)=0 .
$$

The Green's function of the corresponding homogeneous boundary value problem is

$$
G(t, y)= \begin{cases}s(t-1), & 0 \leq s \leq t \leq 1 \\ t(s-1), & 0<t<s \leq 1\end{cases}
$$

The estimate of Green's function is $\Gamma=\frac{1}{4}$.

Suppose that $N=1$, then

$$
M=\left|-\pi^{2}-\arctan (1+32 \sin \pi t)\right| \approx 11.41 .
$$

The inequality (4.22) does not hold since

$$
\Gamma \cdot M \approx 0.25 * 11.41 \approx 2.85>1=N .
$$

The inequality

$$
\frac{1}{4}\left|-\pi^{2} N-\arctan (N+160)\right| \leq N
$$

is not satisfied. Using this method we cannot prove that the boundary value problem (4.24) is solvable.

Example 6. $g(x)=-\sin x$. Consider the boundary value problem

$$
x^{\prime \prime}=-\pi^{2} x-\sin x, x(0)=x(1)=0 .
$$

Denote $u(t)=4 \sin \pi t$. Introduce the new variable $y=x-u(t)$. Therefore $x=$ $y+u(t)$.

Analogously as in the previous example we define

$$
\begin{aligned}
f_{1}(t, x) & =g(t, x)-u^{\prime \prime}(t)=-\pi^{2} x-\sin x+4 \pi^{2} \sin \pi t, \\
F_{1}(t, y) & =f_{1}(t, u(t)+y) \\
& =-\pi^{2}(y+4 \sin \pi t)-\sin (y+4 \sin \pi t)+4 \pi^{2} \sin \pi t \\
& =-\pi^{2} y-\sin (y+4 \sin \pi t), \\
F(t, y) & =F_{1}(t, \delta(-N, y, N)) \\
& =-\pi^{2} \delta(-N, y, N)-\sin (\delta(-N, y, N)+4 \sin \pi t),
\end{aligned}
$$

where $\delta(-N, y, N)$ is the "cut off" function.

Notice that

$$
\max \{|F(t, y)|: 0 \leq t \leq 1,|y| \leq N\}=M(N) .
$$

Consider the homogeneous boundary value problem (4.25) with the Green's function (4.26).

The estimate of Green's function is $\Gamma=\frac{1}{4}$. 
Suppose that $N=0.1$, then

$$
M=\left|-0.1 \pi^{2}-\sin (0.1+4 \sin \pi t)\right| \approx 0.17 .
$$

The inequality

$$
\Gamma \cdot M \approx 0.25 * 0.17 \approx 0.04<0.1=N
$$

holds, therefore the resonant boundary value problem (4.27) has a solution.

The inequality

$$
\frac{1}{4}\left|-\pi^{2} N-\sin (N+4)\right| \leq N
$$

is valid, if $N \leq 0.14$, then the boundary value problem (4.27) has a solution $\mid x(t)-$ $u(t) \mid \leq N$.

Example 7. $g(x)=-\left(e^{x}-1\right)$.

Consider the boundary value problem

$$
x^{\prime \prime}=-\pi^{2} x-\left(e^{x}-1\right), x(0)=x(1)=0 .
$$

Denote $u(t)=32000 \sin \pi t$. Introduce the new variable $y=x-u(t)$. Then $x=$ $y+u(t)$.

Analogously we define

$$
\begin{aligned}
f_{1}(t, x) & =g(t, x)-u^{\prime \prime}(t)=-\pi^{2} x-\left(e^{x}-1\right)-32000 \pi^{2} \sin \pi t, \\
F_{1}(t, y) & =f_{1}(t, u(t)+y) \\
& =-\pi^{2}(y-32000 \sin \pi t)-\left(e^{y-32000 \sin \pi t}-1\right)-32000 \pi^{2} \sin \pi t \\
& =-\pi^{2} y-\left(e^{y-32000 \sin \pi t}-1\right), \\
F(t, y) & =F_{1}(t, \delta(-N, y, N)) \\
& =-\pi^{2} \delta(-N, y, N)-\left(e^{\delta(-N, y, N)-32000 \sin \pi t}-1\right),
\end{aligned}
$$

where $\delta(-N, y, N)$ is the "cut off" function.

Notice that

$$
\max \{|F(t, y)|: 0 \leq t \leq 1,|y| \leq N\}=M(N) .
$$

Consider the homogeneous boundary value problem (4.25) with the Green's function (4.26).

The estimate of the Green's function is $\Gamma=\frac{1}{4}$.

Suppose that $N=0.15$, then

$$
M=\left|-0.15 \pi^{2}-\left(e^{0.15-32000 \sin \pi t}-1\right)\right| \approx 0.48 .
$$

The inequality

$$
\Gamma \cdot M \approx 0.25 * 0.48 \approx 0.12<0.15=N
$$

holds, therefore the resonant boundary value problem is solvable. 
The inequality

$$
\frac{1}{4}\left|-\pi^{2} N-\left(e^{N-32000}-1\right)\right| \leq N
$$

is valid, if $N \leq 0.17$, then the boundary value problem (4.28) has a solution $\mid x(t)-$ $u(t) \mid \leq N$.

Finally, consider example concerning equations with the linear part depending on the derivative $x^{\prime}$. Namely, look at the problem

$$
x^{\prime \prime}+2 x^{\prime}+\left(\pi^{2}+1\right) x=\varphi\left(x, x^{\prime}\right), x(0)=0, x(1)=0,
$$

where $\varphi\left(x, x^{\prime}\right)$ will be defined later.

This problem is resonant since the homogeneous boundary value problem

$$
x^{\prime \prime}+2 x^{\prime}+\left(\pi^{2}+1\right) x=0, x(0)=0, x(1)=0
$$

has the nontrivial solution $x_{0}=e^{-t} \sin \pi t$.

Consider the modified problem

$$
x^{\prime \prime}+2 x^{\prime}+\left(1+\pi^{2}\right) x+\pi x-2 \pi x^{\prime}=\varphi\left(x, x^{\prime}\right)+\pi x-2 \pi x^{\prime},
$$

where the linear part is no more resonant with respect to the boundary conditions $x(0)=x(1)=0$. We will need the Green's function

$$
G(t, s)= \begin{cases}\frac{e^{(1-\pi)(s-t)} \sin (\sqrt{3 \pi} s) \sin (\sqrt{3 \pi}(t-1))}{\sqrt{3 \pi} \sin \sqrt{3 \pi}}, & 0 \leq s \leq t \leq 1, \\ \frac{e^{(1-\pi)(s-t)} \sin (\sqrt{3 \pi}(s-1)) \sin (\sqrt{3 \pi} t)}{\sqrt{3 \pi} \sin \sqrt{3 \pi}}, & 0<t<s \leq 1 .\end{cases}
$$

and

$$
\frac{\partial G(t, s)}{\partial t}=\left\{\begin{array}{l}
\frac{e^{(1-\pi)(s-t)} \sin (\sqrt{3 \pi} s)((\pi-1) \sin (\sqrt{3 \pi}(t-1))+\sqrt{3 \pi} \cos (\sqrt{3 \pi}(t-1)))}{\sqrt{3 \pi} \sin \sqrt{3 \pi}}, \\
\frac{e^{(1-\pi)(s-t)} \sin (\sqrt{3 \pi}(s-1))((\pi-1) \sin (\sqrt{3 \pi} t)+\sqrt{3 \pi} \cos \sqrt{3 \pi} t)}{\sqrt{3 \pi} \sin \sqrt{3 \pi}} .
\end{array}\right.
$$

Computations accomplished by Wolfram Mathematica shows that

$$
|G(t, s)|<7 \text { and }\left|\frac{\partial G}{\partial t}(t, s)\right|<50 \text {. }
$$

Let $\Gamma=7$ and $\Gamma_{1}=50$. We wish to define $N \leq 0$ and $N_{1} \leq 0$ that

$$
\begin{aligned}
& \Gamma \cdot M \leq N, \\
& \Gamma_{1} \cdot M \leq N_{1},
\end{aligned}
$$

where

$$
M=\max _{|x| \leq N,\left|x^{\prime}\right| \leq N_{1}}\left\{\left|\varphi\left(x, x^{\prime}\right)+\pi x-2 \pi x^{\prime}\right|\right\} .
$$


Let $N=7, N_{1}=50$. Let $\varphi\left(x, x^{\prime}\right)$ be a function such that

$$
M=\max _{|x| \leq 7,\left|x^{\prime}\right| \leq 50}\left\{\left|\varphi\left(x, x^{\prime}\right)+\pi x-2 \pi x^{\prime}\right|\right\}
$$

satisfies (4.32). Then the problem

$$
\begin{aligned}
x^{\prime \prime}+(2-2 \pi) x^{\prime}+\left(\pi^{2}+\pi+1\right) x= & \varphi\left(\delta(-7, x, 7), \delta\left(-50, x^{\prime}, 50\right)\right)+\pi \delta(-7, x, 7) \\
& -2 \pi \delta\left(-50, x^{\prime}, 50\right), x(0)=x(1)=0
\end{aligned}
$$

has a solution $x(t)$ such that

$$
\begin{aligned}
& |x(t)|=\left|\int_{0}^{1} G(t, s) F\left(s, x(s), x^{\prime}(s)\right) d s\right| \leq \Gamma \cdot M \leq N, \\
& \left|x^{\prime}(t)\right|=\left|\int_{o}^{1} \frac{\partial G(t, s)}{\partial t} F\left(s, x(s), x^{\prime}(s)\right) d s\right| \leq \Gamma_{1} \cdot M \leq N_{1},
\end{aligned}
$$

where $F$ is the right side of the equation in (4.33), and $x(t)$ is therefore a solution of (4.29). Any continuous function $\varphi\left(x, x^{\prime}\right)$ such that

$$
M=\max _{|x| \leq N,\left|x^{\prime}\right| \leq N_{1}}\left\{\left|\varphi\left(x, x^{\prime}\right)+\pi x-2 \pi x^{\prime}\right|\right\}
$$

satisfy

$$
M \leq \frac{N}{\Gamma}=1 \text { and } M \leq \frac{N_{1}}{\Gamma_{1}}=1
$$

is suitable for (4.29).

\section{CONCLUSION}

If the second order BVP is known to have a solution of type $i$, then the problem can be reduced to a quasi-linear problem "around" a solution, irrespectively of either the original problem is resonant or not.

If the second order formally resonant BVP has not a solution of indefinite type then either it has not a solution at all or it can be reduced to quasi-linear problem.

It is reasonable to combine the "shift" type arguments with multiple different (with different linear parts) quasi-linearizations of a given problem since multiple solutions can be obtained.

Using the "shift" arguments when making quasi-linearization of a resonant problem it is advisable to approximate the nonlinearity by its Taylor approximation.

If an approximation of the expected solution is known then the process of quasilinearization can be made easier and more effective. 


\section{REFERENCES}

[1] A. Almansour and P. Eloe, "Fixed points and solutions of boundary value problems at resonance." Ann. Polon. Math., vol. 115, no. 3, pp. 263-274, 2015, doi: 10.4064/ap115-3-5.

[2] R. Conti, "Equazioni differenziali ordinarie quasilineari con condizioni lineari." Ann. mat. pura ed appl., vol. 57, no. 1, pp. 41-61, 1962, doi: 10.1007/BF02417726.

[3] M. Dobkevich, F. Sadyrbaev, N. Sveikate, and I. Yermachenko, "On Types of Solutions of the Second Order Nonlinear Boundary Value Problems.” Abstract and Applied Analysis, vol. 2014, Article ID 594931, 9 pages, 2014, doi: 10.1155/2014/594931.

[4] X. Han, "Positive solutions for a three-point boundary value problems at resonance." J. Math. Anal. Appl., vol. 336, no. 1, pp. 556-568, 2007, doi: 10.1016/j.jmaa.2007.02.069.

[5] G. Infante, P. Pietramala, and F. A. F. Tojo, "Non-trivial solutions of local and non-local neumann boundary-value problems." Proceedings of the Royal Society of Edinburgh: Section A Mathematics, vol. 146, no. 2, pp. 337-369, 2016, doi: 10.1017/S0308210515000499.

[6] L. K. Jackson and K. W. Schrader, "Comparison Theorems for Nonlinear Differential Equations." J. Diff. Equations, vol. 3, no. 2, pp. 248-255, 1967, doi: 10.1016/0022-0396(67)90029-0.

[7] H. W. Knobloch, "Comparison Theorems for Nonlinear Second Order Differential Equations." J. of Diff. Equations, vol. 1, no. 1, pp. 1-26, 1965, doi: 10.1016/0022-0396(65)90025-2.

[8] H. W. Knobloch, "Second order differential inequalities and a nonlinear boundary value problem." J. of Diff. Equations, vol. 5, no. 1, pp. 55-71, 1969, doi: 10.1016/0022-0396(69)90104-1.

[9] F. Sadyrbaev, "Oscillatory solutions of boundary value problems." In: Springer Proceedings in Mathematics and Statistics. Differential and Difference Equations with Applications (Editors: Sandra Pinelas, Ondřej Došỳ, Zuzana Došlá and Peter Kloeden): ICDDEA, Amadora, Portugal, p. May 2015 (in press), May 2015.

[10] F. Sadyrbaev and I. Yermachenko, "Quasilinearization and multiple solutions of the EmdenFowler type equation." Mathematical Modelling and Analysis., vol. 10, no. 1, pp. 41-50, 2005, doi: 10.1080/13926292.2005.9637269.

[11] F. Sadyrbaev and I. Yermachenko, "Types of solutions and multiplicity results for two-point nonlinear boundary value problems.” Nonlinear Anal., Theory Methods Appl., Ser. A, Theory Methods (Proc. Fourth World Congress of Nonlinear Analysts, Orlando, FL, USA, July 2004), vol. 63, no. 5-7, pp. e1725-e1735, 2005, doi: 10.1016/j.na.2004.12.035.

[12] N. Sveikate, "Resonant problems by quasilinearization." Int. J. Differ. Equ., vol. 2014, Article ID 564914, 8 pages, 2014, doi: 10.1155/2014/564914.

[13] J. R. L. Webb and M. Zima, "Multiple positive solutions of resonant and non-resonant nonlocal boundary value problems." Nonlinear Anal., Theory Methods Appl., Ser. A, Theory Methods, vol. 71, no. 3-4, pp. 1369-1378, 2009, doi: 10.1016/j.na.2008.12.010.

Authors' addresses

N. Sveikate

Daugavpils University, Department of Mathematics, Parades str. 1, LV-5401 Daugavpils, Latvia

E-mail address: nsveikate@inbox. Iv

F. Sadyrbaev

Institute of Mathematics, University of Latvia, Rainis boul. 29, LV-1459 Riga, Latvia

E-mail address: felix@latnet. Iv 